Ciência Florestal, Santa Maria, v. 25, n. 3, p. 721-730, jul.-set., 2015

ISSN 0103-9954

\title{
PAINEL AGLOMERADO DE RESÍDUOS AGROINDUSTRIAIS
}

\author{
PARTICLEBOARDS OF AGROINDUSTRIAL WASTES
}

\author{
Julio Cesar Machado Cravo ${ }^{1}$ Diogo de Lucca Sartori ${ }^{2}$ Juliano Fiorelli ${ }^{3}$ \\ Julio Cesar de Carvalho Balieiro ${ }^{4}$ Holmer Savastano Junior ${ }^{5}$
}

\begin{abstract}
RESUMO
O presente trabalho teve como objetivo desenvolver painéis de partículas aglomeradas de baixa densidade com casca de amendoim, fibra da casca do coco-verde (híbrido) e resina poliuretana bicomponente à base de óleo de mamona. Os painéis experimentais foram fabricados com partículas de até $8 \mathrm{~mm}$ de comprimento e $15 \%$ de resina poliuretana. A prensagem foi realizada a 5,0 MPa, por 10 minutos, a uma temperatura de $100^{\circ} \mathrm{C}$. Foram determinadas as propriedades físico-mecânicas do material, seguindo as recomendações da norma ABNT 14810:2006. Os resultados demonstraram que o desempenho mecânico que atende às recomendações estabelecidas pela normativa ANSI A208.1-1999 e ABNT 14.810-2:2006.
\end{abstract}

Palavras-chave: fibra de coco; casca de amendoim; resina poliuretana; painéis aglomerados.

\begin{abstract}
The present study aimed to develop particleboards of low density with peanut shell, coconut husk fiber green (hybrid) and two-component polyurethane resin based on castor oil. The experimental panels were manufactured with particles up to $8 \mathrm{~mm}$ and $15 \%$ polyurethane resin. The pressing was carried out at 5.0 $\mathrm{MPa}$ for 10 minutes at $100^{\circ} \mathrm{C}$ of temperature. The physical-mechanical properties of the material were determined as recommended by ABNT 14810:2006. The results demonstrated a mechanical performance that meets the recommendations established by normative ANSI A208.1-1999 and ABNT 14.810-2:2006.

Keywords: coconut fiber; peanut hulls; polyurethane resin; particleboard
\end{abstract}

\section{INTRODUÇÃO}

Os painéis de madeira aglomerada surgiram na Alemanha no início da década de 40, como alternativa para viabilizar a utilização de resíduo de madeira, face à dificuldade de obtenção de madeiras de boa qualidade para produção de painéis compensados, devido ao isolamento da Alemanha durante a Segunda Guerra Mundial (IWAKIRI, 2005).

No Brasil, as produções desses painéis tiveram início nos anos 60 , com a utilização de

1 Zootecnista, Doutorando em Qualidade e Produtividade Animal, Departamento de Biossistemas, Faculdade de Zootecnia e Engenharia de Alimentos, Av. Duque de Caxias Norte, 225, CEP 13635-900, Pirassununga (SP), Brasil. juliomachadomachado@hotmail.com

2 Zootecnista, Doutorando em Qualidade e Produtividade Animal, Departamento de Biossistemas, Faculdade de Zootecnia e Engenharia de Alimentos, Av. Duque de Caxias Norte, 225, CEP 13635-900, Pirassununga (SP), Brasil. luccasartori@hotmail.com

3 Engenheiro Civil, Dr., Professor do Departamento de Engenharia Biossistemas, Faculdade de Zootecnia e Engenharia de Alimentos, Av. Duque de Caxias Norte, 225, CEP 13635-900, Pirassununga (SP), Brasil. julianofiorelli@usp.br

4 Zootecnista, Dr., Professor do Departamento de Ciências Básicas, Faculdade de Zootecnia e Engenharia de Alimentos, Av. Duque de Caxias Norte, 225, CEP 13635-900, Pirassununga (SP), Brasil. balieiro@usp.br

5 Engenheiro Civil, Dr., Professor do Departamento de Engenharia Biossistemas, Faculdade de Zootecnia e Engenharia de Alimentos, Av. Duque de Caxias Norte, 225, CEP 13635-900, Pirassununga (SP), Brasil. holmersj@usp.br

Recebido para publicação em 31/07/2012 e aceito em 21/10/2013 
prensas cíclicas, sendo questionados quanto a sua qualidade, pois se alegava que esses tipos de painéis apresentavam alta absorção de água e inchamento em espessura (MELO, 2009).

Entretanto, a partir dos anos 90, as empresas brasileiras que fabricavam painéis aglomerados começaram a investir em tecnologia, resultando na substituição das prensas cíclicas pelas prensas contínuas. $\mathrm{O}$ aperfeiçoamento adquirido no processo de fabricação dos aglomerados, pelas empresas brasileiras, em anos posteriores, resultou no aprimoramento da qualidade desse produto, culminando para a expansão do consumo desses painéis no mercado nacional (MATTOS et al., 2008).

No entanto, segundo Guler et al. (2007), a demanda por madeira pela indústria florestal tem aumentado anualmente, culminando para o declínio das florestas naturais. Como alternativa, as indústrias florestais têm buscado diferentes fontes lignocelulósicas, como matéria-prima para produção de painéis. De acordo com Rowell et al. (2000) os painéis aglomerados também podem ser fabricados a partir de qualquer material lignocelulósico que lhes confiram resistência mecânica e peso específico pré-estabelecido, já que a composição química dos materiais lignocelulósico é semelhante à da madeira, mais precisamente com a das madeiras duras, que contêm menor teor de lignina e maior teor de hemiceluloses do tipo pentosanas.

Segundo Mendes et al. (2010), a produção de painéis aglomerados a partir de resíduos agroindustriais proporciona agregar valor ao resíduo e, além disso, poderá atender à crescente demanda da indústria de painéis de madeira, além de possibilitar sua expansão, diminuir a utilização de madeira e, consequentemente, a pressão sobre as florestas, e ainda reduzir custos de produção dos painéis, tornando-os ainda mais competitivos no cenário econômico.

Mendes et al. (2010) estudaram o efeito da incorporação de casca de café $(25,50$ e $75 \%)$ nas propriedades físico-mecânicas de painéis aglomerados à base de Eucalyptus urophylla. Os autores diagnosticaram redução nas propriedades físico-mecânicas com aumento do teor de casca de café. Guler et al. (2007) avaliaram diferentes níveis $(0,25,50,75$ e $100 \%)$ de incorporação de casca de amendoim (Arachis hypoqaea) em painéis aglomerado de Pinus nigra Arnold. Os autores constataram baixo desempenho das propriedades físico-mecânicas com o aumento dos níveis de casca de amendoim, sendo que apenas o nível de $25 \%$ atendeu ao recomendado pela norma europeia EN 312 (2003).

Bektas et al. (2005) estudaram a influência do caule do girassol (Helianthus annuus) nas propriedades físico-mecânicas de painéis aglomerados de madeira (Populus alba). Nesse estudo, os autores testaram a adição de $0,25,50,75$ e $100 \%$ de caule de girassol. Os autores encontraram decréscimo nas propriedades físico-mecânicas, proporcional ao aumento dos níveis de caule de girassol.

Colli et al. (2010) avaliaram as propriedades físico-mecânicas de painéis fabricados com partículas de madeira (Schyzolobium amazonicum Huber ex. Ducke) a diferentes proporções de fibra de coco (Cocos nucifera L.) e resina (6 e $8 \%$ de ureia-formaldeído). Os autores diagnosticaram melhoras significativas para o Módulo de Ruptura e Resistência à Tração, quando incorporada a fibra de coco às partículas de madeira. Para os níveis de resina, os autores constataram melhor desempenho físico-mecânico para os painéis que possuíam $8 \%$ de resina.

Guler et al. (2007) avaliaram o desempenho físico-mecânico de painéis de partículas de densidade $700 \mathrm{~kg} / \mathrm{m}^{3}$ de casca de amendoim e madeira $(0$, $25,50,75$ e $100 \%$ ) aglomeradas com resina ureiaformaldeído. Os autores constataram diferenças $(\mathrm{P}<0,05)$ nos valores de MOR e MOE entre os painéis de $100 \%$ casca de amendoim (MOR - 9,90 MPa; MOE - 1276,76 MPa) com os painéis de 25\% casca de amendoim e $75 \%$ partículas de madeira (MOR - 14,10 MPa; MOE - 1885,00 MPa).

Fiorelli et al. (2012) avaliaram painéis aglomerados de alta densidade de fibra da casca do coco-verde e resina poliuretana bi-componente à base de óleo de mamona. Segundo os autores, os painéis de partículas produzidos com fibra da casca do coco-verde, apresentaram desempenho físicomecânico acima dos limites mínimos recomendados pelos principais documentos normativos nacionais e internacionais.

Diante do exposto, esse trabalho teve como objetivo estudar o comportamento físico-mecânico de painéis aglomerados de baixa densidade com casca de amendoim, fibra da casca do coco e resina poliuretana à base de óleo de mamona. A incorporação da fibra do coco-verde na casca de amendoim visa maximizar as propriedades físicomecânicas do compósito. 


\section{MATERIAL E MÉTODOS}

\section{Obtenção das matérias-primas}

Foram produzidos painéis particulados de baixa densidade com massa específica nominal de $500 \mathrm{~kg} / \mathrm{m}^{3}$ (ANSI A208.1-1999) com $20 \mathrm{~mm}$ de espessura. Os resíduos agroindustriais utilizados para confecção dos painéis experimentais foram adquiridos de duas indústrias do estado de São Paulo. A casca de amendoim (Arachis hypoqaea) foi obtida de uma indústria proveniente da cidade de Tupã, enquanto que a fibra de coco (Cocos nucifera) foi proveniente da cidade de Marília, ambas situadas na região oeste do estado de São Paulo.

O adesivo empregado para confecção dos painéis à base dos resíduos agroindustriais supracitados foi a resina poliuretana à base de óleo de mamona. Esse material foi adquirido da empresa Plural Química - São Carlos-SP.

\section{Fabricação dos painéis experimentais de baixa densidade}

A casca de amendoim e a fibra de coco foram dispostas em bandejas e secas em uma estufa modelo MA035, com temperatura de $60^{\circ} \mathrm{C}$, até atingir o teor de umidade de $3 \%$. Após o período de secagem, a casca de amendoim e a fibra da casca do coco foram levadas a um moinho de facas modelo MA683/3 com malha de $8 \mathrm{~mm}$ (SAMPATHRAJAN et al., 1991), sendo posteriormente pesadas em uma balança eletrônica modelo AY220 para averiguar a massa total necessária para produzir os painéis particulados com densidade de $500 \mathrm{~kg} / \mathrm{m}^{3}$ (ANSI A208.1-1999).

Posteriormente, as partículas foram levadas a um misturador planetário modelo MT120, no qual foi adicionado adesivo poliuretano à base de óleo de mamona, com proporção de $15 \%$ do peso seco da matéria-prima.

Finalizada a mistura entre a matéria-prima e a resina, o material foi acondicionado em uma caixa formadora de painel e remetido para uma prensa termo-hidráulica com temperatura média de $100^{\circ} \mathrm{C}$, por aproximadamente 10 minutos, com pressão média de $5 \mathrm{MPa}$.

Após a saída da prensa, os painéis ficaram empilhados por 72 horas, período em que ocorre a continuação do processo de cura da resina. No total, foram produzidos seis painéis experimentais, sendo três painéis de casca de amendoim (CA) e três painéis híbridos, com casca de amendoim e fibra da casca do coco (50\% casca de amendoim e 50\% fibra de coco). A Figura 1 ilustra os painéis experimentais.
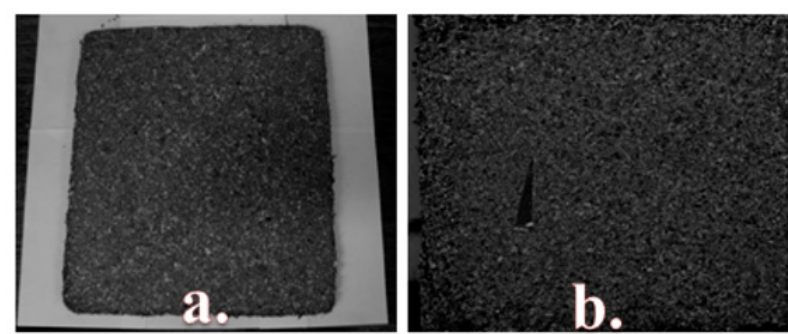

FIGURA1: Painéis experimentais. (a) painel híbrido. (b) painel casca de amendoim.

FIGURE 1: Experimental panels. (a) hybrid panel. (b) peanut Shell panel.

\section{Ensaios realizados}

Após o período de cura dos painéis (72 h), esses foram esquadrejados com auxílio de uma serra elétrica e posteriormente acondicionados em uma câmara climatizada com umidade relativa de $65 \%$ e temperatura $20^{\circ} \mathrm{C}$, até atingirem a umidade de equilíbrio. A Tabela 1 sumariza a quantidade e as dimensões dos corpos de prova, baseadas na norma ABNT 14.810:2006 - Chapa de Madeiras

TABELA 1: Dimensões e quantidade dos corpos de prova por painéis.

TABLE 1: Dimensions and quantity of specimens by panels.

\begin{tabular}{lccc}
\hline \multicolumn{1}{c}{ Ensaio } & Comprimento $(\mathrm{mm})$ & Largura $(\mathrm{mm})$ & Quantidade \\
\hline Densidade Aparente & 50 & 50 & 10 \\
Flexão Estática & 250 & 50 & 10 \\
Tração Perpendicular & 50 & 50 & 10 \\
Absorção 2 e 24 horas & 25 & 25 & 10 \\
Inchamento 2 e 24 horas & 25 & 25 & 10 \\
\hline
\end{tabular}


Aglomeradas, para as seguintes propriedades: densidade aparente (12\% umidade), módulo de ruptura (MOR) e módulo de elasticidade (MOE) na flexão estática, adesão interna (AI), absorção (ABS) e inchamento (INCH) 2 e $24 \mathrm{~h}$.

Os ensaios de flexão e tração perpendicular foram realizados em uma máquina universal de ensaios modelo EMIC DL 30.000 e determinadas as propriedades de MOR, MOE e AI. Para os ensaios de absorção (ABS) e inchamento (INCH), os corpos de prova foram introduzidos em um recipiente com água a $20^{\circ} \mathrm{C}$ e ficaram submersos durante o período de 2 e 24 horas, seguindo as recomendações da ABNT 14.810:2006 devido à semelhança dos painéis em estudo com as Chapas de partículas de madeira.

\section{Avaliação microestrutural dos painéis experimentais}

A análise microestrutural de painéis particulados foi realizada com objetivo de avaliar a aglomeração das partículas e justificar as propriedades físico-mecânicas. Tangjuank e Kumfu (2011) utilizaram esse recurso para explicar o aumento na densidade dos painéis em relação à variação nos níveis de resina, possibilitando a discussão das variáveis, absorção, inchamento e condutividade térmica.

Nesse âmbito, foi realizada uma análise microestrutural dos painéis experimentais (casca de amendoim e híbrido) em Microscópio Eletrônico de Varredura (MEV), marca Hitachi modelo Analytical Table Top Microscope TM300.

Quatro corpos de prova de cada painel com dimensões de $10 \mathrm{~mm} \times 10 \mathrm{~mm} \times 10 \mathrm{~mm}$ foram utilizados para a análise qualitativa microestrutural.

\section{Avaliação dos resultados}

Os dados de propriedades físico-mecânica (Densidade Aparente, MOR, MOE, AI, ABS e INCH 2 e $24 \mathrm{~h}$ ) foram submetidos ao Teste de Anderson Darling, para averiguar a normalidade dos resíduos e posteriormente testados pelo Teste de Bartlett, para averiguar a homogeneidade das variâncias. Esses testes foram realizados no software $\mathrm{R}$ versão 2.5.1, com $\mathrm{P}<0,05$. A realização desses testes é necessária, pois de acordo com Nogueira (2007), são pressuposições para realizar a ANOVA.

Posteriormente aos testes de pressuposição da ANOVA foram montados oito experimentos.
Sete experimentos (Densidade Aparente, MOR, MOE, ABS e INCH 2 e $24 \mathrm{~h}$ ) foram dispostos em um delineamento inteiramente casualizado (DIC), em que o fator estudado correspondeu ao painel com dois níveis (Híbrido e CA), totalizando em dois tratamentos (cada experimento). O modelo estatístico adotado para esses experimentos foi:

$$
\begin{aligned}
& \mathrm{Y}_{\mathrm{ijk}}=\mu+\mathrm{t}_{\mathrm{i}}+\mathrm{e}_{\mathrm{ijk}} \\
& \mathrm{Y}_{\mathrm{ijk}}=\mathrm{k} \text {-ésima observação da variável }
\end{aligned}
$$
Densidade Aparente, MOR, MOE, ABS e INCH 2 e 24 h, no i-ésimo nível do fator painel (Híbrido ou CA) na j-ésima repetição; $\mu=$ média geral; $t_{i}=$ efeito do i-ésimo nível de fator painel, i = Híbrido e CA; $\mathrm{e}_{\mathrm{ijk}}=$ efeito residual.

Já para variável Adesão Interna (AI), o experimento foi montado segundo o delineamento em blocos casualizados (DBC), nos quais a fonte de variação carga de ruptura foi o controle local (Bloco) imposto. O fator estudado correspondeu ao painel com dois níveis (Híbrido e CA), totalizando em dois tratamentos. Segundo Nogueira (2007), a utilização de Blocos (controle local) no experimento é uma forma de minimizar o erro experimental, neutralizando o efeito local nos dados obtidos, devido à presença de uma heterogeneidade no ambiente ou material experimental. Com relação a esse experimento, o modelo estatístico empregado foi:

$$
\mathrm{Y}_{\mathrm{ij}}=\mu+\mathrm{t}_{\mathrm{i}}+\mathrm{b}_{\mathrm{j}}+\mathrm{e}_{\mathrm{ij}}
$$

$\mathrm{Y}_{\mathrm{ij}}=$ valor observado, da variável AI, referente ao i-ésimo nível do fator painel (Híbrido ou CA) aplicado no j-ésimo bloco; $\mu=$ média geral; $t_{i}=$ efeito do i-ésimo nível de fator painel, $\mathrm{i}=$ Híbrido e CA; $\mathrm{b}_{\mathrm{j}}=$ efeito do j-ésimo bloco; $\mathrm{e}_{\mathrm{ij}}=$ efeito residual.

A variável Densidade Aparente, MOR, MOE, AI, ABS e INCH 2 e 24 horas foram analisados por meio do Teste $\mathrm{F}$ quando a ANOVA foi significativa, sendo testado a $\mathrm{P}<0,05$. O software utilizado para interpretar esse resultado foi $\mathrm{o}$ Programa R versão 2.5.1.

\section{RESULTADOS E DISCUSSÃO}

\section{Pressuposição ANOVA}

A Tabela 2 sumariza os valores de 
probabilidade encontrados nos testes empregados para verificar a normalidade dos dados e homogeneidade das variâncias a $\mathrm{P}<0,05$.

Observa-se na Tabela 2 que não foi constatado homogeneidade $(\mathrm{P}<0,005)$ para variável absorção 2 horas. Como essa pressuposição não foi atendida utilizou-se a Estatística não Paramétrica para analisar essa variável, adotando o Teste de Mann-Whitney. Segundo Fonseca e Martins (1996), esse teste é usado para testar se duas amostras independentes foram retiradas de populações com médias iguais, e não exige nenhuma consideração sobre as distribuições populacionais e suas variâncias.

Para as outras variáveis estudadas (Densidade Aparente, MOR, MOE, AI, ABS 24 h e INCH 2 e $24 \mathrm{~h})$ foram diagnosticadas normalidade $\mathrm{e}$ homogeneidade dos dados $(\mathrm{P}<0,05)$. Como essas pressuposições estiveram adequadas, essas variáveis foram analisadas pela ANOVA.

\section{Análise inferencial}

A Tabela 3 descreve os valores de probabilidade encontrados na análise de variância a $\mathrm{P}<0,05$.

Foi constatada a significância a $\mathrm{P}<0,05$ para as variáveis estudadas, com exceção para a Densidade Aparente (12\% de umidade) e INCH 2 e $24 \mathrm{~h}$ que apresentaram $P$-values superiores a $\mathrm{P}<0,05$. Para as variáveis que exibiram $P$-value significativo, os valores médios experimentais foram avaliados pelo Teste $\mathrm{F}$ a $\mathrm{P}<0,05$. Esses resultados estão listados na Tabela 4.

Para variável Densidade Aparente (12\% de umidade) não foi identificado diferenças entre as médias dos painéis experimentais. Esse resultado demonstra maior confiabilidade para avaliação das demais propriedades desse estudo (MOR, MOE e AI), uma vez que Melo et al. (2010) e Iwakiri et al. (2008) diagnosticaram elevados coeficientes de correlação entre a densidade e propriedades mecânicas. A Figura 2 sumariza a comparação entre as Densidades Aparentes para os painéis experimentais, amendoim (CA) e híbrido.

Observa-se na Figura 2 que os painéis experimentais produzidos com densidade nominal

TABELA 2: P-values pressuposição da ANOVA.

TABLE 2: $P$-values assumption of ANOVA.

\begin{tabular}{ccc}
\hline \multirow{2}{*}{ Densidade Aparente (12\% de umidade) } & Anderson Darling & Bartlett \\
\cline { 2 - 3 } & $0,853121002^{\mathrm{NS}}$ & $0,167700367^{\mathrm{NS}}$ \\
\hline MOR & $0,29543764^{\mathrm{NS}}$ & $0,39743641^{\mathrm{NS}}$ \\
MOE & $0,51564663^{\mathrm{NS}}$ & $0,07746570^{\mathrm{NS}}$ \\
AI & $0,227967852^{\mathrm{NS}}$ & $0,078978675^{\mathrm{NS}}$ \\
ABS 2 h & $0,814807238^{\mathrm{NS}}$ & $0,019445995^{*}$ \\
ABS 24 h & $0,870709572^{\mathrm{NS}}$ & $0,094294909^{\mathrm{NS}}$ \\
INCH 2 h & $0,685387978^{\mathrm{NS}}$ & $0,564533112^{\mathrm{NS}}$ \\
INCH 24 h & $0,641274222^{\mathrm{NS}}$ & $0,204841677^{\mathrm{NS}}$ \\
\hline
\end{tabular}

Em que: ${ }^{\mathrm{NS}}=$ Não significativo a $\mathrm{P}<0,05 ; *=$ Significativo a $\mathrm{P}<0,05$.

TABELA 3: $P$-values da ANOVA.

TABLE 3: $P$-values of ANOVA.

\begin{tabular}{cc}
\hline Densidade Aparente $(12 \%$ de umidade $)$ & Tratamento \\
\cline { 2 - 2 } MOR & $0,0558^{\mathrm{NS}}$ \\
\hline MOE & $0,00000^{* *}$ \\
AI & $0,00000^{* *}$ \\
ABS 24 h & $0,0035^{*}$ \\
INCH 2 h & $0,0024^{*}$ \\
INCH 24 h & $0,6709^{\mathrm{NS}}$ \\
\hline
\end{tabular}

Em que: ${ }^{\mathrm{NS}}=$ Não significativo a $\mathrm{P}<0,05 ; *$ = Significativo a $\mathrm{P}<0,05 ; * *$ Significativo a $\mathrm{P}<0,05$. 
TABELA 4: Valores médios experimentais.

TABLE 4: Experimental mean.

\begin{tabular}{ccccc}
\hline Variável & Painel Híbrido & $\mathrm{CV}(\%)$ & Amendoim $(\mathrm{CA})$ & $\mathrm{CV}(\%)$ \\
\hline Densidade Aparente $\left(\mathrm{kg} / \mathrm{m}^{3}\right)$ & $503,04 \mathrm{~A}$ & 8,09 & $542,06 \mathrm{~A}$ & 5,85 \\
MOR $(\mathrm{MPa})$ & $3,88 \mathrm{~A}$ & 7,67 & $1,82 \mathrm{~B}$ & 21,88 \\
MOE $(\mathrm{MPa})$ & $445,2 \mathrm{~A}$ & 5,12 & $333,8 \mathrm{~B}$ & 21,1 \\
AI (MPa) & $0,2563 \mathrm{~A}$ & 49,32 & $0,1590 \mathrm{~B}$ & 37,79 \\
ABS 24 h (\%) & $68,33 \mathrm{~A}$ & 13,87 & $61,13 \mathrm{~B}$ & 4,95 \\
INCH 2 h (\%) & $8,18 \mathrm{~A}$ & 12,52 & $8,36 \mathrm{~A}$ & 10,04 \\
INCH 24 h (\%) & $18,1 \mathrm{~A}$ & 5,42 & $18,92 \mathrm{~A}$ & 8,82 \\
\hline
\end{tabular}

Em que: Médias com letras maiúscula diferentes na linha diferem pelo Teste $\mathrm{F}$ a $\mathrm{P}<0,05$

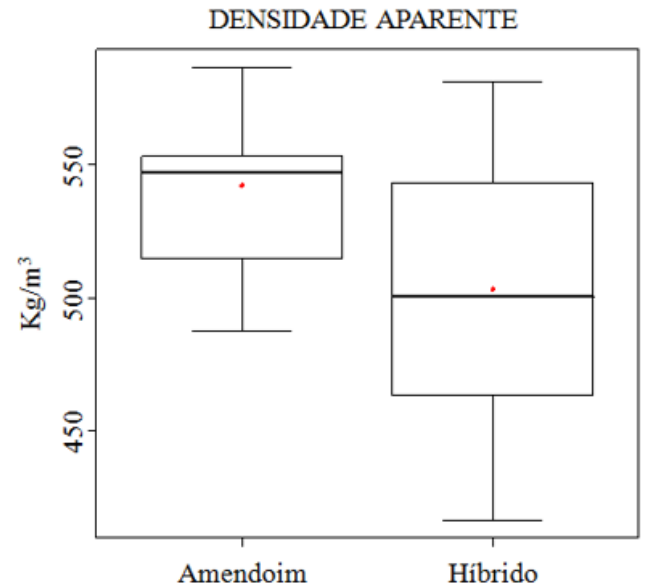

FIGURA 2: Comparação dos conjuntos de dados dos painéis experimentais.

FIGURE 2: Comparison on the data sets of experimental panels.

de $500 \mathrm{~kg} / \mathrm{m}^{3}$, apresentaram variação na densidade. Além disso, nota-se que o painel experimental de amendoim apresentou menor dispersão dos dados, podendo ser constatado pelo tamanho da cauda. Esse comportamento pode estar atrelado à diferença na geometria da fibra de coco e da casca de amendoim, à espessura da microfibrila, ao teor de umidade e à falta de homogeneidade de distribuição das partículas no colchão.

De acordo com a norma ANSI A208.1-1999, painéis particulados de baixa densidade devem apresentar densidade menor que $640 \mathrm{~kg} / \mathrm{m}^{3}$. Com base nos valores médios obtidos nesse trabalho, pode-ser afirmar que os painéis experimentais são de baixa densidade.

Os valores médios de MOR e MOE do painel híbrido foram significativamente $(\mathrm{P}<0,05)$ superiores àqueles obtidos para painel de CA (Tabela 4), com 53,09\% e 25,02\%, respectivamente. A diferença significativa diagnosticada nesse experimento (MOR) pode ser explicada pela afirmação de Panyakaew e Fotios (2011). Segundo esses autores, o MOR não só depende da força de ligação entre as fibras, mas também da força individual da fibra, de sua geometria e espessura.

Outro fator que pode ter influenciado no desempenho do MOR é a característica química da casca de amendoim e da fibra de coco. Segundo Passos (2005), a celulose é um polissacarídeo linear e o principal constituinte da parede celular das fibras vegetais, sendo responsável pela estabilidade e resistência das fibras. Além da celulose, a lignina é outro polímero que fornece resistência à fibra. Essas são informações devem ser investigadas, pois não foram averiguadas nesse estudo.

Para variável AI, observa-se, na Tabela 4 que o painel híbrido exibiu valor médio experimental significativamente $(\mathrm{P}<0,05)$ superior ao painel amendoim (CA), equivalendo a $37,89 \%$ de diferença. A geometria da fibra de coco pode ser uma das razões que levaram ao aumento significativo dessa variável, uma vez que essa deve ter contribuído para melhor dispersão da resina sobre os painéis, proporcionando maior aderência entres as partículas de casca de amendoim. Outro fator que deve ser destacado é a porosidade da superfície da fibra de coco-verde, o que garante melhor distribuição da resina e uma adesão mecânica entre as partículas.

Para ABS $24 \mathrm{~h}$, o painel CA apresentou valor médio experimental inferior $(\mathrm{P}<0,05)$ ao painel híbrido, o equivalente a $10,53 \%$ (Tabela 4 ). A diferença entre a geometria das partículas de fibra de coco e casca de amendoim é uma hipótese que pode 
explicar a maior absorção de água do painel híbrido, pois se infere que a adição das fibras de coco criou espaços vazios no painel. De acordo com Tangjuank (2011) e Tangjuank e Kumfu (2011), painéis com maior número de espaços vazios absorvem mais água.

Embora tenha constatado maior ABS 24 h para o painel híbrido, não foi diagnosticada diferença $(\mathrm{P}<0,05)$ entre os painéis experimentais para a variável INCH 2 e $24 \mathrm{~h}$. Isso demonstra que a adição de $50 \%$ de fibra de coco ao painel CA não influencia essa propriedade.

A Tabela 5 descreve os valores do Teste de Mann-Whitney para variável AB $2 \mathrm{~h}$.

TABELA 5: Teste de Mann-Whitney - Absorção $2 \mathrm{~h}$ TABLE 5: Mann-Whitney-Absorption $2 \mathrm{~h}$

\begin{tabular}{cc}
\hline Ztab & Zcal \\
\hline$-1,96<$ Zcal $<1,96$ & $-8,14^{*}$ \\
\hline
\end{tabular}

Em que: $*=$ Significativo a $\mathrm{P}<0,05$

Para o período das 2 horas foi diagnosticado diferença significativa $(\mathrm{P}<0,05)$ para o Teste de Mann-Whitney (Tabela 5), demonstrando que as médias experimentais dos painéis são diferentes. Com base nesse teste, pode-se afirmar que o painel híbrido apresentou maior $(\mathrm{P}<0,05)$ absorção que o painel de casca de amendoim, no período das 2 horas. Esses valores estão descritos na Tabela 6.

TABELA 6: Médias experimentais - Absorção 2 horas.

TABLE 6: Experimental means - Absorption 2 hours.

\begin{tabular}{ccc}
\hline Painel & Absorção 2 horas (\%) & CV(\%) \\
\hline Híbrido & $28,82 \mathrm{~A}$ & 6,36 \\
Amendoim (CA) & $21,27 \mathrm{~B}$ & 3,71 \\
\hline
\end{tabular}

Em que: Médias com letras maiúscula diferentes na coluna diferem pelo Teste Mann-Whitney a $\mathrm{P}<0,05$

Apesar da incorporação da fibra de coco à casca de amendoim (painel híbrido) ter proporcionado aumento significativo no MOR, MOE e AI, essa adição resultou no aumento significativo da absorção de água (Tabelas 5 e 6). Embora não se tenha efetuado ensaio de envelhecimento, inferese que o painel híbrido poderia apresentar maior degradação natural, uma vez que a umidade é um dos fatores que contribui para reduzir a durabilidade do material (GONÇALEZ et al., 2010). Além disso, a alta absorção de água pode culminar no aparecimento de micro-organismos, pois, segundo Highley (1999), o teor de umidade de $30 \%$ ou mais é necessário para o crescimento de fungos.

\section{Análise comparativa entre 0 desempenho físico-mecânico dos painéis experimentais com alguns painéis particulados de baixa densidade}

A Tabela 7 apresenta valores de MOR, MOE e AI dos painéis experimentais (Híbrido e CA), valores preconizados pela ANSI A208.1:1999, ABNT 14810:2006 (painéis com espessura de 14$20 \mathrm{~mm}$ ) e valores de trabalhos experimentais de painéis particulados de baixa densidade à base de resíduos lignocelulósicos.

$\mathrm{O}$ valor médio experimental (MOR) do painel híbrido ficou acima do limite inferior preconizado pela ANSI A208.1:1999 e abaixo do estipulado pela ABNT 14810:2006. Porém, em comparação com outros painéis derivados de resíduos agroindustriais, o painel híbrido foi superior ao Kenaf e casca de coco. Para o painel de CA, o valor médio do MOR ficou abaixo do encontrado por Vijayaraghavan e Swaminathan (1992). Esse resultado pode ter relação com o tipo de resina utilizada (ureia-formaldeído) e com os parâmetros de processamento do painel (temperatura de $170^{\circ} \mathrm{C}$ ). Com relação ao MOE, os painéis de CA e Híbrido apresentaram valores abaixo do estipulado pela norma ANSI A208.1-1999. Para o painel híbrido, observa-se que o MOE foi superior ao Kenaf e casca de coco. Já o painel experimental à base de casca de amendoim foi superior apenas ao Kenaf (Tabela 7).

$\mathrm{O}$ valor médio de AI dos painéis de CA e Híbrido foi superior ao estipulado pela ANSI A208.1-1999 e inferior às recomendações da norma NBR 14810:2006. Esse resultado é explicado, pois as normas em questão foram elaboradas para painéis de partículas de madeira e utilizadas nesse estudo como um parâmetro de comparação devido à semelhança entre os materiais.

A Tabela 8 descreve a comparação dos valores de ABS e INCH $2 \mathrm{~h}$ e 24 h dos painéis de CA e Híbrido e de alguns trabalhos sobre painéis particulados de baixa densidade à base de resíduos.

Os valores médios experimentais de absorção obtidos para os painéis em estudo foram inferiores aos extraídos da literatura (Tabela 8). Duas hipóteses 
TABELA 7: Comparação entre os valores médios de MOR, MOE e AI - experimentais e literatura.

TABLE 7: Comparison between mean values of MOR and MOE - experimental and literature.

\begin{tabular}{|c|c|c|c|c|c|}
\hline & Densidade $\left(\mathrm{kg} / \mathrm{m}^{3}\right)$ & MOR (MPa) & MOE (MPa) & AI (MPa) & Fonte \\
\hline Híbrido & 500 & 3,88 & 445,2 & 0,26 & \multirow{2}{*}{ Estudo } \\
\hline $\mathrm{CA}$ & 500 & 1,82 & 333,8 & 0,16 & \\
\hline Norma ANSI A208.1-1999 & $>640$ & 3 & 550 & 0,10 & ANSI A208.1:1999 \\
\hline Norma ABNT 14810:2006 & ----- & 16 & ----- & 0,35 & ABNT 14810:2006 \\
\hline $\begin{array}{l}\text { Casca de Amendoim } \\
\text { (Arachis hypoqaea) }\end{array}$ & 540 & 6,3 & 523 & ----- & $\begin{array}{c}\text { Vijayaraghavan e Swaminathan } \\
\text { (1992) }\end{array}$ \\
\hline $\begin{array}{l}\text { Kenaf } \\
\text { (Hibiscus cannabinus) }\end{array}$ & 200 & 1,1 & 300 & 0,10 & Xu et al. (2004) \\
\hline $\begin{array}{l}\text { Casca de coco } \\
\text { (Cocos nucifera). }\end{array}$ & 450 & 1,94 & 365 & 0,002 & \multirow{2}{*}{ Panyakaew e Fotios (2011) } \\
\hline $\begin{array}{l}\text { Bagaço de Cana } \\
\text { (Sacharum officinarum) }\end{array}$ & 450 & 4,16 & 957 & 0,014 & \\
\hline
\end{tabular}

TABELA 8: Valores médios de absorção e inchamento experimental e literatura

TABLE 8: Values of absorption and swelling experimental literature

\begin{tabular}{|c|c|c|c|c|c|c|}
\hline \multirow{2}{*}{ Painel } & \multirow{2}{*}{ Densidade $\left(\mathrm{kg} / \mathrm{m}^{3}\right)$} & \multicolumn{2}{|c|}{ ABS (\%) } & \multicolumn{2}{|c|}{ INCH (\%) } & \multirow{2}{*}{ Fonte } \\
\hline & & $2 \mathrm{~h}$ & $24 \mathrm{~h}$ & $2 \mathrm{~h}$ & $24 \mathrm{~h}$ & \\
\hline Híbrido & 500 & 28,82 & 68,33 & 8,18 & 18,1 & \multirow{2}{*}{ Estudo } \\
\hline Amendoim (CA) & 500 & 21,27 & 61,13 & 8,36 & 18,92 & \\
\hline $\begin{array}{l}\text { Fibra de Papiro } \\
\text { (Typha angustifólia) }\end{array}$ & 266 & 101 & 120 & 24 & 33 & Tangjuank e Kumfu (2011) \\
\hline $\begin{array}{l}\text { Folha de Abacaxi } \\
\text { (Annas comosus) }\end{array}$ & 232 & 190 & 250 & 20 & 27 & Tangjuank (2011) \\
\hline
\end{tabular}

podem explicar essa diferença. A primeira tem relação com a quantidade e tipo de resina empregada na fabricação dos painéis, pois, segundo Tangjuank e Kumfu (2011), painéis fabricados com maiores níveis de resina apresentam menor absorção de água, pois a resina cura nos espaços vazios, resultando em menor porosidade do painel. A segunda hipótese tem relação com a densidade e a porosidade do painel, pois, segundo Tangjuank (2011), painéis de baixa densidade apresentam maior absorção de água, devido à característica porosa do material.

Para variável inchamento, os painéis híbridos e de CA apresentaram valores médios inferiores aos encontrados na literatura. Esse comportamento pode estar atrelado a vários fatores, segundo Guler et al. (2007), o inchamento dos painéis depende da ineficiência do volume e distribuição da resina, compatibilidade da resina com as partículas, umidade insuficiente, composição química etc.

\section{Análise microestrutural dos painéis experimentais}

A Figura 3 apresenta imagens de MEV para painéis híbridos e de CA com ampliação de 100x e 200x, respectivamente.

Observa-se na Figura 3 que no painel híbrido, as fibras de coco-verde ficaram sobrepostas às partículas da casca de amendoim, resultando em maior número de espaço vazios em relação ao painel de CA. Essa característica tem relação com as diferenças de geometria das partículas (fibra de coco - lamelar e casca de amendoim - disco).

Os espaços vazios observados nas imagens de MEV explicam a porosidade dos painéis híbridos e justificam os maiores valores de absorção descritos.

\section{CONCLUSÃO}

Pelos resultados apresentados, concluise: O painel de CA apresentou valores de MOR e 

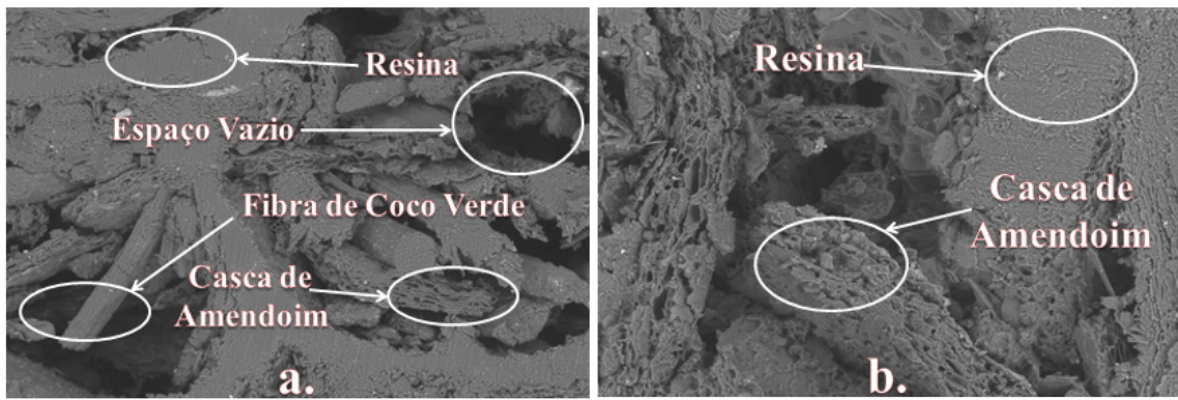

FIGURA 3: Imagem dos painéis experimentais. (a) painel híbrido (100x) e (b) painel casca de amendoim $(200 x)$.

FIGURE 3. Image of experimental panels. (a) hybrid panel (100x) and (b) peanut shell panel (200x).

MOE abaixo do estabelecido pela norma ANSI A208.1:1999, enquanto a variável AI ficou acima do preconizado por essa norma demonstrando eficiência da resina poliuretana na aderência das partículas;

A adição de fibra da casca do coco-verde à casca de amendoim (painel híbrido) proporcionou aumento significativo nas propriedades de MOR, MOE e AI, no entanto, as propriedades físicas foram afetadas, devido ao aumento da porosidade do painel;

A resina poliuretana à base de óleo de mamona apresentou desempenho adequado e pode ser utilizada como substituta para a resina comercial à base de ureia-formaldeído;

As características dos painéis em estudo permitem indicar sua aplicação como isolante térmico acústico, no entanto, ensaios específicos devem ser realizados no material para verificar sua eficiência quando aplicado nessas condições.

\section{AGRADECIMENTOS}

Os autores agradecem à FAPESP, CNPq e FINEP pelo apoio concedido à pesquisa.

\section{REFERÊNCIAS BIBLIOGRÁFICAS}

ABNT 14810 (2006). Chapas de madeira aglomerada- parte 3: Métodos de Ensaio, terminologia. Rio de Janeiro, $32 \mathrm{p}$.

ANSI A208.1(1999). American National Standard Particleboard. $13 \mathrm{p}$.

BEKTAS, I. et al. The manufacture of particleboards using sunflower stalks (Helianthus annuus L.) and poplar wood (Populus alba L.). J. Compos. Mater, Lancaster, v. 39, n. 5, p. 467-473, 2005.
COLLI, A. et al. Propriedades de chapas fabricadas com partículas de madeira de paricá (Schyzolobium amazonicum) e fibra de coco verde (Cocos nucifera). Revista Árvore, Viçosa, v. 34, n. 2, p. 333 - 338, 2010.

FIORELLI, J. et al. Particulate composite based on coconut fiber and castor oil polyurethane adhesive: An ecoefficient product. Industrial Crops and Products, v. 40, p. 69-75, 2012.

FONSECA, J. S. ; MARTINS, G. A. Curso de Estatística. São Paulo: Atlas,1996. 320 p.

GULER, C.; COPUR, Y.; TASCIOGLU, C. The manufacture of particleboards using mixture of peanut (Arachis hypoqaea) and European Black pine (Pinus nigra Arnold) wood chips. Bioressource Technology, v. 99, p. 2893-2897, 2007.

GONÇALEZ, J. C. et al. Efeito da radiação ultravioleta na cor da madeira de freijó (Cordia goeldiana) após receber produtos de acabamento. Ciência Florestal, Santa Maria, v. 20, n. 4, p. 657-664, 2010.

HIGHLEY, T. Biodeterioration of wood. Wood Handbook: Wood as an Engineering Material [M]. Wisconsin: USDA Forest Service Forest Products Laboratory, 1999. p.16.

IWAKIRI, S. et al. Influência da massa específica sobre as propriedades mecânicas de painéis aglomerados. Floresta, Curitiba, v. 38, n. 3, p. 487 $-493,2008$.

IWAKIRI, S. et al. Produção de painéis de madeira aglomerada de Grevillea robusta A. Cunn. ex R. Br. Revista Árvore, Viçosa, v. 28, n. 6, p. 883-887, 2004.

IWAKIRI, S. Painéis de madeira reconstítuida. 1. ed. Curitiba: Fundação de Pesquisas Florestais do Paraná, 2005.

MATTOS, R. L. G., GONÇALVES, R. M., 
CHAGAS, F. B. Painéis de madeira Brasil: panorama e perspectivas. BNDS Setorial v. 27, p. 121-156, 2008.

MELO, R. R. et al. Avaliação das propriedades físico-mecânicas de painéis aglomerados de Eucalyptus grandis colados com ureia-formaldeído e tanino-formaldeído. Floresta, Curitiba, v. 40, n. 3, p. $497-506,2010$.

MELO, R. R. et al. Propriedades físicomecânicas de painéis aglomerados produzidos com diferentes proporções de madeira e casa de arroz. Ciência Florestal, Santa Maria, v. 19, n. 4, p. 449-460, 2009.

MENDES, R. F. et al. Efeito da incorporação de casca de café nas propriedades físico-mecânicas de painéis aglomerados de Eucalyptus urophylla $\mathrm{S}$. T. Blake. Revista Ciênc. Agrotec., Lavras, v. 34, n. 3, p. $610-617,2010$.

MENDES, R. F. et al. Painéis aglomerados com bagaço de cana em associação com madeira de eucalipto. Scientia Forestalis, Piracicaba, v. 38, n. 86, p. $285-295,2010$.

NOGUEIRA, M. C. S. Experimentação Agronômica I. Piracicaba, 2007.

PANYAKAEW, S. ; FOTIOS, S. New thermal insulation boards made from coconut husk and bagasse. Energy and Buildings, v. 43, n. 7, p.1732-1739, 2011.

PASSOS, P. R. A. Destinação sustentável de cascas de coco (cocos nucifera) verde: obtenção de telhas e chapas de partículas. 2005. 186 f. Tese (Doutorado em Engenharia) Universidade Federal do Rio de Janeiro, Rio de Janeiro, 2005.

ROWELL R. M.; HAN J. S.; ROWELL J. S. Characterization and Factors Affecting Fiber Properties. In: NATURAL POLYMERS AND AGROFIBERS BASED COMPOSITES, 2000, São Carlos. Anais... São Carlos: Embrapa Instrumentação Agropecuária, 2000.

SAMPATHRAJAN, A.; VIJAYARAGHAVAN, N. C.; SWAMINATHAN, K. R. Mechanical and thermal properties of particle boards made from farm residues. Bioresource Technology, v. 40, p. 249-251, 1991.

TANGJUANK, S. ; KUMFU, S. Particle boards from papyrus fibers as thermal insulation. Journal of Applied Sciences, v. 11, n. 14, p. 2640-2645, 2011.

TANGJUANK, S. Thermal insulation and physical properties of particleboards from pineapple leaves. International Journal of Physical Sciences, v. 6, n. 19, p. 4528-4532, 2011.

$\mathrm{XU}$, J. et al. Manufacture and properties of lowdensity binderless particleboard from kenaf core. J. Wood Sci., v. 50, p. 62-67, 2004.

VAN DAM, J.E.G. et al. Process for production of high density/high performance binderless boards from whole coconut husk. Industrial Crops and Products, v.19, p. 207-216, 2004. 